Tohoku J. Exp. Med., 2008, 216, 53-59

\title{
The Non-steroidal Anti-inflammatory Drugs Protect Mouse Cochlea against Acoustic Injury
}

\author{
Tomofumi Hoshino, ${ }^{1}$ KeijI Tabuchi,,${ }^{1}$ Yuki Hirose, ${ }^{1}$ Isao Uemaetomari, ${ }^{1}$ \\ Hidekazu Murashita, ${ }^{1}$ Tadamichi Tobita ${ }^{1}$ and Akira Hara ${ }^{1}$ \\ ${ }^{1}$ Department of Otolaryngology, Graduate School of Comprehensive Human Sciences, University \\ of Tsukuba, Tsukuba, Japan
}

\begin{abstract}
Acoustic injury is a common cause of hearing loss for people in industrial societies. Cyclooxygenase (COX) and lipoxygenase (LOX) are two important enzymes involved in arachidonic acid metabolism. Two COX isozymes are characterized, COX-1 and COX-2, that differ in terms of regulatory mechanisms of expression. Although COX-1, COX-2, and LOX are expressed in cochlea, their roles played in cochlear acoustic injury have not fully been evaluated. Non-steroidal anti-inflammatory drugs (NSAIDs) inhibit either COX or LOX, or both. This study evaluated the effects of NSAIDs on the functional recovery of the cochlea after acoustic injury. Mice were exposed to a 4-kHz pure tone of $128 \mathrm{~dB}$ SPL (sound pressure level) for 4 hours and received one of the following drugs for two weeks after acoustic overexposure: indomethacin (COX-1 inhibitor), meloxicam, SC58125, and CAY10404 (COX-2 inhibitors), and nordihydroguaiaretic acid (LOX inhibitor). The hearing ability was evaluated using an auditory brainstem response (ABR) before and after overexposure. The ABR threshold shifts, defined as subtraction between ABR thresholds before and after overexposure, were compared among the control and the medication groups at one and two weeks after acoustic overexposure. Treatment of mice with either indomethacin or nordihydroguaiaretic acid decreased the ABR threshold shifts after overexposure, indicating that COX-1 and LOX inhibitors exhibited protective effects against acoustic injury. In contrast, COX-2 inhibitors, meloxicam, SC58125, and CAY10404, showed no noticeable effects on the ABR threshold shifts. These findings suggest that COX-1 and LOX are involved in the pathogenesis of acoustic injury in cochlea. — cyclooxygenase; lipoxygenase; non-steroidal anti-inflammatory drugs; cochlea; acoustic injury.
\end{abstract}

Tohoku J. Exp. Med., 2008, 216 (1), 53-59.

(C) 2008 Tohoku University Medical Press

Exposure to high levels of sound pressure causes hearing loss by damaging the cochlea, particularly inducing los of sensory hair cells (reviewed by Tabuchi et al., 2007). Acoustic injury is one of the main sources of hearing loss for people in industrial societies. In addition, the noisy sounds of everyday life make acoustic injury a predicament that may affect all members of society.

Prostaglandins (PGs) and leukotrienes (LTs) are generated through the arachidonic cascade. In the first step of the arachidonic cascade, arachi-

Received May 12, 2008; revision accepted for publication July 16, 2008.

Correspondence: Keiji Tabuchi, Department of Otolaryngology, Graduate School of Comprehensive Human Sciences, University of Tsukuba, 1-1-1 Tennodai, Tsukuba, 305-8575, Japan.

e-mail: ktabuchi@md.tsukuba.ac.jp 
donic acid (AA) is generated from membrane phospholipid precursors by phospholipase A2. The next stage of this cascade has two main pathways: the cyclooxygenase (COX) pathway that produces PGs and thromboxanes (TXs), and the lipoxygenase (LOX) pathway that produces LTs.

$\mathrm{COX}$ is currently categorized into two isozymes, COX-1 and COX-2 (Gierse et al. 1996; Gilroy et al. 1998; Vane et al. 1998; Reddy et al. 1999). COX-1 is constitutively expressed in most tissues of mammals, whereas COX-2 is not detected in normal tissues with the exception of the kidney and central nervous system (LopezGarcia and Laird 1998), and is induced in response to various stimuli, such as cytokines, bacterial lipopolysaccharide and growth factors. (Barnett et al. 1994; Blanco et al. 1999)

Non-steroidal anti-inflammatory drugs (NSAIDs) block the COX and/or LOX pathways as competitive inhibitors. COX inhibitors and LOX inhibitors inhibit the production of PGs and LTs, respectively. PGs and/or LTs are known to be inflammatory and pyretogenic substances, and thus some of NSAIDs are clinically used as analgesics and antipyretics. Although NSAIDs are widely used clinically, the effects of NSAIDs on acoustic injury of the cochlea have attracted only little attention. To the best of our knowledge, only the effects of diclofenac (Lamm and Arnold 1998) and salicylate (Kopke et al. 2000) on acoustic injury have been reported.

In this study, we examined the effects of NSAIDs on acoustic injury of the cochlea. We used selective inhibitors of COX-1, COX-2 and LOX. Indomethacin is a COX-1 preferential inhibitor (Barnett et al. 1994; Blanco et al. 1999). Meloxicam (Pairet et al. 1998; Blanco et al. 1999), SC58125 (Anderson et al. 1996) and CAY 10404 (Katada et al. 2005) are defined as COX-2 inhibitors. Nordihydroguaiaretic acid (NDGA) was reported to be a selective LOX inhibitor (Mayer et al. 1986). The purpose of the present study was therefore to examine the effects of these NSAIDs on acoustic injury of the mouse cochlea and to clarify differences in contribution to the protective effect.

\section{Materials ANd Methods}

Animals

Sixty ddY mice ( 8 weeks of age) were used in this study. The ddY mouse is one of the inbred strain mice that was established at the National Institute of Health of Japan. This strain is easy to maintain because of good fertility and growth. Therefore, it is frequently used as a subject for pharmacological and toxicological experiment in Japan. The animals were kept on a normal day/ night cycle with free access to food. The care and use of the animals were approved by the Animal Experiment Committee of the University of Tsukuba.

\section{Auditory brainstem response (ABR) testing}

Mice were anesthetized with an intraperitoneal injection of pentobarbital sodium $(50 \mathrm{mg} / \mathrm{kg}$ body weight). Positive, negative and ground electrodes were inserted subcutaneously at the vertex, mastoid and back, respectively. Pure tone bursts (rise and fall times, $1 \mathrm{~ms}$; duration, $10 \mathrm{~ms}$ ) were used to evoke ABR. Evoked responses were amplified, filtered with a band pass of 200 to $3,000 \mathrm{~Hz}$ and averaged with 1,000 sweeps using a signal processor (Synax 1200, NEC, Tokyo, Japan). Sound intensity varied in $5 \mathrm{~dB}$ steps. The hearing threshold was defined as the lowest intensity yielding the consistent appearance of at least one ABR peak. ABR threshold shifts from pre-exposure levels were examined one and two weeks after acoustic overexposure.

\section{Acoustic overexposure}

The mice were subjected to a $4 \mathrm{kHz}$ pure tone of $128 \mathrm{~dB}$ SPL for 4 hours through an open field system inside a sound-exposure chamber (Type 4212, Brüel \& Kjaer, Copenhagen, Denmark). Sound intensity was measured at multiple locations within the sound chamber using an SPL meter (NA-60, RION, Tokyo, Japan) to ensure the uniformity of exposure stimuli, and the sound intensity of exposure stimuli was confirmed to vary by a maximum of $1 \mathrm{~dB}$ (Uemaetomari et al. 2005; Murashita et al. 2006).

\section{Histological examination}

The mice were sacrificed under deep anesthesia two weeks after acoustic overexposure. Cardiac perfusion was performed with $4 \%$ paraformaldehyde in $0.1 \mathrm{M}$ phosphate-buffered saline. Cochleae were then removed and immersed in the same fixative for 8 hours. After fixation, whole mounts of the organ of Corti were dissected as surface preparations, and the nuclei of hair cells were 
stained with propidium iodide (PI, Molecular Probes Inc., OR).

All specimens were thoroughly inspected under a laser confocal microscope (TCS 4D, Leica Microsystems, Wetzlar, Germany). The number of missing hair cells (absence of PI staining) was determined from the apex to the base in $0.33 \mathrm{~mm}$ segments and plotted as cytocochleograms.

\section{Drug administration}

Indomethacin, SC58125, CAY10404 and NDGA (Cayman chemical, Ann Arbor, MI) were dissolved in peanut oil $(5 \mathrm{ml} / \mathrm{kg}$ ), and meloxicam (BIOMOL, Plymouth Meeting, PA) in physiological saline solution (PSS, $5 \mathrm{ml} / \mathrm{kg}$ ). All drugs were administered subcutaneously in the back of the animals. Drug administration started immediately after acoustic overexposure and was then administered twice a day during the two-week experimental period. Control mice received $5 \mathrm{ml} / \mathrm{kg}$ of PSS or peanut oil at the same time and schedule after acoustic overexposure. The concentration of each drug was decided based on the minimum concentration at which the drug inhibits PGE2 activity or pain. (Nakayama et al. 1998; Pairet et al. 1998; Katada et al. 2005)

\section{Experimental design}

Mice were randomly assigned to one of the following 7 treatment groups:

(1) indomethacin group $(n=10)$ : Mice in this group were treated with $5 \mathrm{mg} / \mathrm{kg}(n=5)$ or $1 \mathrm{mg} / \mathrm{kg}(n=$ 5) indomethacin.

(2) meloxicam group $(n=10)$ : Mice in this group were treated with $50 \mathrm{mg} / \mathrm{kg}(n=5)$ or $10 \mathrm{mg} / \mathrm{kg}$ $(n=5)$ meloxicam.

(3) SC58125 group $(n=5)$ : Mice in this group were treated with $10 \mathrm{mg} / \mathrm{kg} \mathrm{SC} 58125$.

(4) CAY10404 group $(n=15)$ : Mice in this group were treated with $30 \mathrm{mg} / \mathrm{kg}(n=5), 10 \mathrm{mg} / \mathrm{kg}(n=$ $5)$ or $3 \mathrm{mg} / \mathrm{kg}(n=5)$ CAY 10404 .

(5) NDGA group $(n=10)$ : Mice in this group were treated with $100 \mathrm{mg} / \mathrm{kg}(n=5)$ or $10 \mathrm{mg} / \mathrm{kg}(n=5)$ NDGA.

(6) PSS-treated control group $(n=5)$ : Mice in this group were treated with $5 \mathrm{ml} / \mathrm{kg}$ PSS.

(7) peanut oil-treated control group $(n=5)$ : Mice in this group were treated with $5 \mathrm{ml} / \mathrm{kg}$ peanut oil.

\section{Data analysis}

All values are presented as mean \pm standard deviation (S.D.) ABR threshold shifts. Percent of missing hair cells were evaluated using two-way factorial ANOVA. Scheffe test and Fisher's PLSD test were used as a post hoc test. A $p$ value of less than 0.05 was considered significant.

\section{Results}

ABR threshold shift after acoustic overexposure

The effect of indomethacin, a COX-1 inhibitor, on ABR threshold shifts one and two weeks after acoustic overexposure is shown in Fig. $1 \mathrm{~A}-\mathrm{B}$. Indomethacin $(5 \mathrm{mg} / \mathrm{kg})$ significantly reduced the ABR threshold shift as compared with peanut oil-given control animals both one and two weeks after acoustic overexposure $(p<$ 0.01 ), although indomethacin at a lower concentration $(1 \mathrm{mg} / \mathrm{kg})$ failed to alleviate acoustic injury at one or two weeks after acoustic overexposure $(p>0.05$ at one and two weeks). As shown in Fig. 1C-D, NDGA (100 mg/kg), a LOX selective inhibitor, significantly decreased the ABR threshold shift both one and two weeks after acoustic overexposure (two-way factorial ANOVA: $p<$ 0.01 , Scheffe test: $p<0.01$ in the $100 \mathrm{mg} / \mathrm{kg}$ group and $p>0.05$ in the $10 \mathrm{mg} / \mathrm{kg}$ group).

The effects of meloxicam, SC58125 and CAY10404, COX-2 inhibitors, on the ABR threshold shifts induced by acoustic overexposure are shown in Fig. 2A-B, respectively. Meloxicam $(50 \mathrm{mg} / \mathrm{kg})$ slightly reduced the ABR threshold shift one week after acoustic overexposure (Fig. 2A: $p=0.04$ ); however, there was no statistically significant difference in the ABR threshold shift between the meloxicam groups (10 or $50 \mathrm{mg} / \mathrm{kg}$ ) and the peanut oil-given control group at two weeks after acoustic overexposure (Fig. 2B). As shown in Fig. 2C-F, SC58125 and CAY10404, which are more selective $\mathrm{COX}-2$ inhibitors than meloxicam, did not have any effect on the ABR threshold shift at one or two weeks after acoustic overexposure.

\section{Histological change by medication}

Our previous reports clarified that acoustic 
T. Hoshino et al.
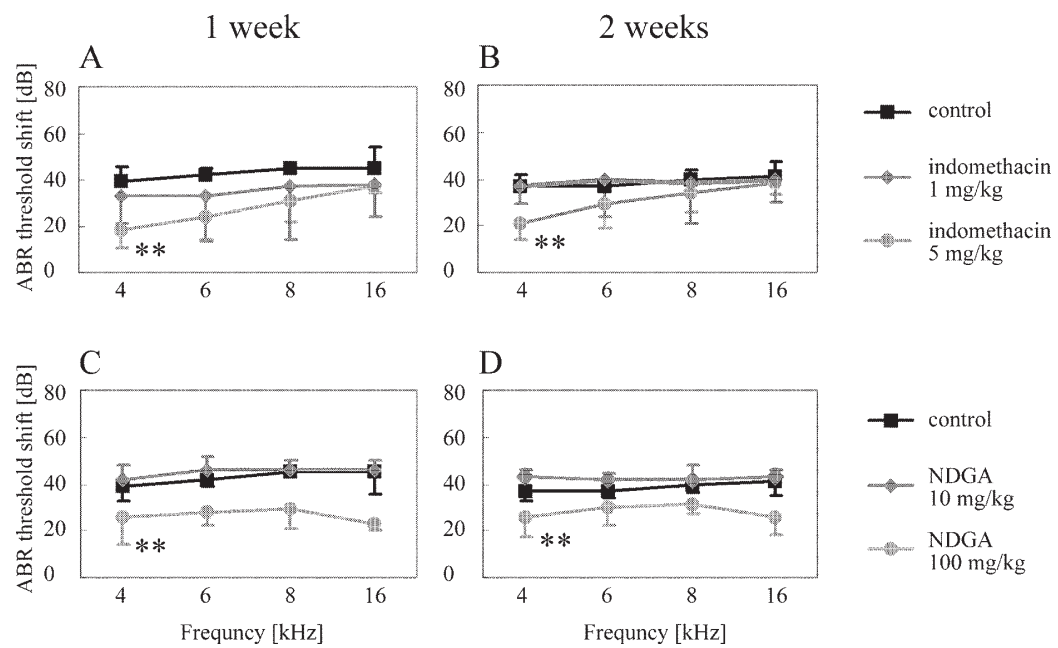

Fig. 1. The effects of COX-1 and LOX inhibitors on ABR threshold shifts.

ABR threshold shifts in the indomethacin group (A and B) and NDGA group (C and D) one week (A and C) and two weeks (B and D) after acoustic overexposure. The COX-1 and LOX inhibitors decreased ABR threshold shifts. Data was analyzed using a two-way factorial ANOVA, Scheffe test, $* * p<0.01$.
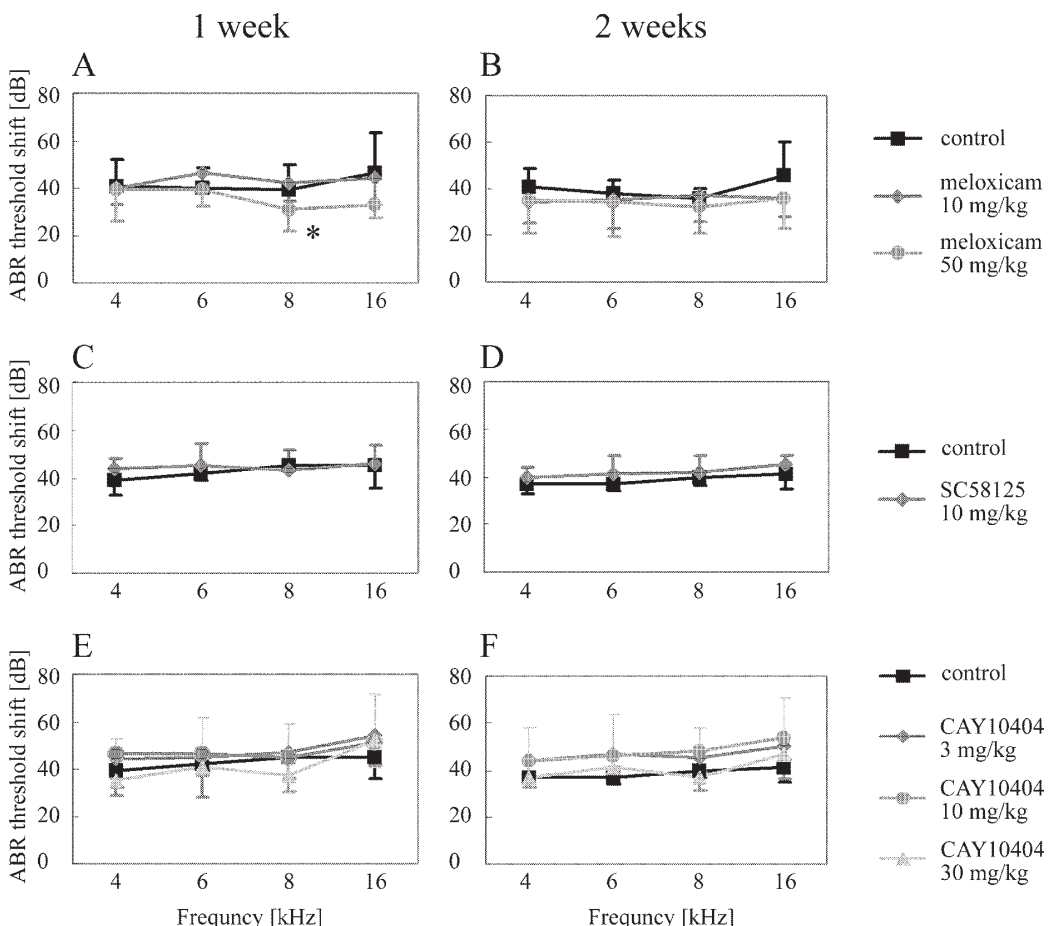

Fig. 2. The effects of COX-2 inhibitors on ABR threshold shifts.

ABR threshold shifts in the meloxicam group (A and B), SC58125 group (C and D), and CAY10404 group (E and F) one week (A, C, and E) and two weeks (B, D, and F) after acoustic overexposure. The COX-2 inhibitors did not decrease ABR threshold shifts Data was analyzed using a two-way factorial ANOVA, Scheffe test, ${ }^{*} p<0.05$. 
A
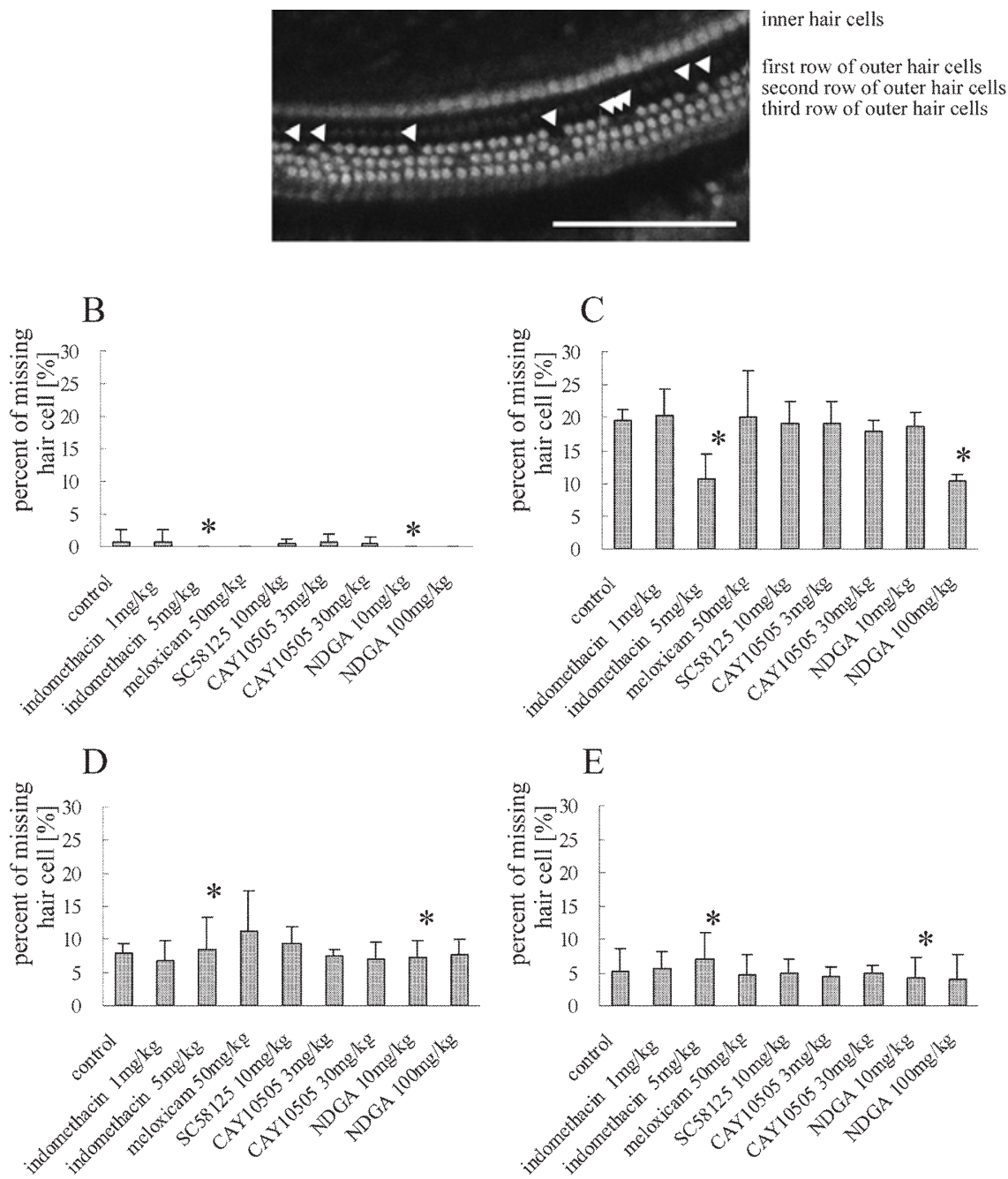

Fig. 3. Profile of hair cell loss after acoustic overexposure.

Propidium iodide staining of the nuclei of hair cells (A) Representative micrograph of organ of Corti in $66 \%$ region from the apex. Note that acoustic overexposure induced hair cell loss as indicated by arrowheads (absence of staining of propidium iodide). Graphs showing hair cell loss in a $66 \%$ region from the apex by medication, $(\mathrm{B})$ inner hair cells, (C) first row of outer hair cells, (D) second row of outer hair cells, and (E) third row of outer hair cells. Analysis of data was performed using a two-way factorial ANOVA, Fisher's PLSD, $* p<0.05$. Scale bar: $0.1 \mathrm{~mm}$.

overexposure of $128 \mathrm{~dB}$ SPL for 4 hours induced hearing loss and hair cell loss (Uemaetomari et al. 2005; Murashita et al. 2006). The maximum hair cell loss was observed in a $66 \%$ region from apex of the cochlea in this model (Fig. 3A: Uemaetomari et al. 2005; Murashita et al. 2006). Fig. 3 shows the percentages of hair cell loss in this region (B-E). Indomethacin $(5 \mathrm{mg} / \mathrm{kg})$ and NDGA $(100 \mathrm{mg} / \mathrm{kg})$ significantly reduced the hair cell loss compared with the control group ( $p<$ 0.05 ), indicating that COX-1 and LOX inhibitors have the protective effect against acoustic injury.

\section{Discussion}

To our knowledge, diclofenac (Lamm and Arnold 1998) and salicylate (Kopke et al. 2000) are the only two NSAIDs reported to protect the cochlea against acoustic injury. Although they are 
COX-1 inhibitors, it has not been reported whether other NSAIDs, COX-2 and LOX inhibitors, also protect against acoustic injury. As NSAIDs are capable of blocking the COX and LOX pathways, the protective mechanism of the cochlea against acoustic injury might differ among the various categories of NSAIDs. We, therefore, tested several kinds of NSAIDs in acoustically injured mice and compared their protective effect. The present study demonstrated that indomethacin and NDGA also have protective effects on acoustic injury of the cochlea. Considering the selectivity of the drugs used in the present study, it is highly likely that COX-1 and LOX are involved in acoustic injury of the cochlea.

Although little is known about the mechanism of cochlea protection by NSAIDs, it is possible that NSAIDs have anti-inflammatory and anti-oxidant actions. As regards the anti-inflammatory action of NSAIDs, TXs and LTs are generated via COX and LOX pathways, respectively. Over-production of TXs and LTs is reported to be toxic to the cochlea (Jung et al. 1992; Umemura 1993); and it is possible that NSAIDs protect the cochlea against acoustic injury by inhibiting overproduction of these metabolites. Regarding the anti-oxidant action, accumulated evidence suggests that reactive oxygen species (ROS) are produced by acoustic overexposure and that ROS play key roles in acoustic injury (Yamane et al. 1995; Yamasoba et al. 1998; Ohlemiller et al. 1999). Although the mechanisms generating ROS in the cochlea after acoustic overexposure have not been clarified. It has been suggested that xanthine oxidase (Seidman et al. 1993), mitochondria (Chance et al. 1979) and arachidonic cascade (Rieger et al. 2002) are generally associated with the production of ROS. AA is oxidized by COX to form PGG2, which is subsequently transformed by hydroperoxidase to form PGH2. As ROS are produced during the conversion of PGG2 to PGH2 (Yu and Wells 1995), ROS production is inhibited by COX inhibitors. Similarly, LOX oxidizes AA to form hydroperoxy-eicosatetraenoic acid (HPETE). Subsequently, HPETE is converted to hydroxy-eicosatetraenoic acid (HETE) by hydroperoxidase, and again ROS are produced during the conversion of HPETE to HETE (Yu and Wells 1995). COX and LOX inhibitors thus block ROS production and these actions might account for the protective mechanism of COX and LOX inhibitors.

\section{Conclusion}

The present study demonstrates the protective effects of indomethacin and NDGA against acoustic injury, suggesting that COX-1 and LOX play key roles in this type of injury. Given that NSAIDs are widely used in humans, these antiinflammatory drugs could be used as a potential and new treatment strategy for cochlear injury.

\section{Acknowledgment}

Funding for the work was through Grants-inAid for Scientific Research (C) 20591969 and for Young Scientists (B)18791186 and 19791190 from the Ministry of Education, Culture, Sports, Science and Technology of Japan. We thank Dr. V-S Bhinu (AAFC-Canada) for his assistance in manuscript preparation.

\section{References}

Anderson, G.D., Hauser, S.D., McGarity, K.L., Bremer, M.E., Isakson, P.C. \& Gregory, S.A. (1996) Selective inhibition of cyclooxygenase (COX)-2 reverses inflammation and expression of COX-2 and interleukin 6 in rat adjuvant arthritis. J. Clin. Invest., 97, 2672-2679.

Barnett, J., Chow, J., Ives, D., Chiou, M., Mackenzie, R., Osen, E., Nguyen, B., Tsing, S., Bach, C., Freire, J., Chan, H., Sigal, E. \& Ramesha, C. (1994) Purification, characterization and selective inhibition of human prostaglandin $\mathrm{G} / \mathrm{H}$ synthase 1 and 2 expressed in the baculovirus system. Biochim. Biophys. Acta, 1209, 130-139.

Blanco, F.J., Guitian, R., Moreno, J., de Toro, F.J. \& Galdo, F. (1999) Effect of antiinflammatory drugs on COX-1 and COX-2 activity in human articular chondrocytes. J. Rheumatol., 26, 1366-1373.

Chance, B., Sies, H. \& Boveris, A. (1979) Hydroperoxide metabolism in mammalian organs. Physiol. Rev., 59, 527-605.

Gierse, J.K., McDonald, J.J., Hauser, S.D., Rangwala, S.H., Koboldt, C.M. \& Seibert, K. (1996) A single amino acid difference between cyclooxygenase-1 (COX-1) and -2 $(\mathrm{COX}-2)$ reverses the selectivity of COX-2 specific inhibitors. J. Biol. Chem., 271, 15810-15814.

Gilroy, D.W., Tomlinson, A. \& Willoughby, D.A. (1998) Differential effects of inhibitors of cyclooxygenase (cyclooxygenase 1 and cyclooxygenase 2 ) in acute inflammation. Eur. J. Pharmacol., 355, 211-217.

Jung, T.T., Park, Y.M., Miller, S.K., Rozehnal, S., Woo, H.Y. \& Baer, W. (1992) Effect of exogenous arachidonic acid metabolites applied on round window membrane on hearing and their levels in the perilymph. Acta Otolaryngol. 
Suppl., 493, 171-176.

Katada, J., Saito, H. \& Ohashi, A. (2005) Significance of cyclooxygenase- 2 induced via p38 mitogen-activated protein kinase in mechanical stimulus-induced peritoneal adhesion in mice. J. Pharmacol. Exp. Ther., 313, 286-292.

Kopke, R.D., Weisskopf, P.A., Boone, J.L., Jackson, R.L., Wester, D.C., Hoffer, M.E., Lambert, D.C., Charon, C.C., Ding, D.L. \& McBride, D. (2000) Reduction of noiseinduced hearing loss using L-NAC and salicylate in the chinchilla. Hear. Res., 149, 138-146.

Lamm, K. \& Arnold, W. (1998) The effect of predonisolone and non-steroidal anti-inflammatory agents on the normal and noise-damaged guinea pig inner ear. Hear. Res., 115, 149-161.

Lopez-Garcia, J.A. \& Laird, J.M. (1998) Central antinociceptive effects of meloxicam on rat spinal cord in vitro. Neuroreport, 9, 647-651.

Mayer, B., Moser, R., Gleispach, H. \& Kukovetz, W.R. (1986) Possible inhibitory function of endogenous 15-hydroperoxyeicosatetraenoic acid on prostacyclin formation in bovine aortic endothelial cells. Biochim. Biophys. Acta., 875, 641-653.

Murashita, H., Tabuchi, K., Hoshino, T., Tsuji, S. \& Hara, A. (2006) The effects of tempol, 3-aminobenzamide and nitric oxide synthase inhibitors on acoustic injury of the mouse cochlea. Hear. Res., 214, 1-6.

Nakayama, M., Uchimura, K., Zhu, R.L., Nagayama, T., Rose, M.E., Stetler, R.A., Isakson, P.C., Chen, J. \& Graham, S.H. (1998) Cyclooxygenase-2 inhibition prevents delayed of CA1 hippocampal neurons following global ischemia. Proc. Natl. Acad. Sci. USA, 95, 10954-10959.

Ohlemiller, K.K., Wright, J.S. \& Dugan, L.L. (1999) Early elevation of cochlear reactive oxygen species following noise exposure. Audiol. Neurootol., 4, 229-236.

Pairet, M., van Ryn, J., Schierok, H., Mauz, A., Trummlitz, G. \& Engelhardt, G. (1998) Differential inhibition of cyclooxygenase- 1 and -2 by meloxicam and its 4 '-isomer. Inflamm. Res., 47, 270-276.

Reddy, S.T., Tiano, H.F., Langenbach, R., Morham, S.G. \&
Herschman, H.R. (1999) Genetic evidence for distinct roles of COX-1 and COX-2 in the immediate and delayed phases of prostaglandin synthesis in mast cells. Biochem. Biophys. Res. Commun., 265, 205-210.

Rieger, J.M., Shah, A.R. \& Gidday, J.M. (2002) Ischemiareperfusion injury of retinal endothelium by cyclooxygenase- and xanthine oxidase- derived superoxide. Exp. Eye Res., 74, 493-501.

Seidman, M.D., Shivapuja, B.G. \& Quirk, W.S. (1993) The protective effects of allopurinol and superoxide dismutase on noise-induced cochlear damage. Otolaryngol. Head Neck Surg., 109, 1052-1056.

Tabuchi, K., Murashita, H. \& Hara, A. (2007) Acoustic injury of the cochlea: The role of reactive oxygen species and mechanisms of hair cell death. Recent advances in Auditory neuroscience, edited by Tabuchi, K. \& Hara, A. Research Signpost, Kerala, India, pp. 11-18.

Uemaetomari, I., Tabuchi, K., Hoshino, T. \& Hara, A. (2005) Protective effect of calcineurin inhibitors on acoustic injury of the cochlea. Hear. Res., 209, 86-90.

Umemura, K., Asai, Y., Uematsu, T. \& Nakashima, M. (1993) Role of thromboxane A2 in a microcirculation disorder of the rat inner ear. Eur. Arch. Otorhinolaryngol., 250, 342-344.

Vane, J.R., Bakhle, Y.S. \& Botting, R.M. (1998) Cyclooxygenase 1 and 2. Annu. Rev. Pharmacol. Toxicol., 38, 97-120.

Yamane, H., Nakai, Y., Takayama, M., Konishi, K., Iguchi, H., Nakagawa, T., Shibata, S., Kato, A., Sunami, K. \& Kawakatsu, C. (1995) The emergence of free radicals after acoustic trauma and strial blood flow. Acta Otolaryngol. Suppl., 519, 87-92.

Yamasoba, T., Nuttall, A.L., Harris, C., Raphael, Y. \& Miller, J.M. (1998) Role of gluthathione in protection against noise-induced hearing loss. Brain Res., 784, 82-90.

Yu, W.K. \& Wells, P.G. (1995) Evidence for lipoxygenasecatalyzed bioactivation of phenytoin to a teratogenic reactive intermediate: in vivo studies using linoleic acid-dependent soybean lipoxygenase, and in vivo studies using pregnant CD-1 mice. Toxicol. Appl. Pharmacol., 131, 1-12. 